\title{
Exercise-induced mitogen-activated protein kinase signalling in skeletal muscle
}

\author{
Yun Chau Long, Ulrika Widegren and Juleen R. Zierath* \\ Department of Surgical Sciences, Section for Integrative Physiology, Karolinska Institutet, \\ von Eulers väg 4, II, SE-171 77 Stockholm, Sweden
}

\begin{abstract}
Exercise training improves glucose homeostasis through enhanced insulin sensitivity in skeletal muscle. Muscle contraction through physical exercise is a physiological stimulus that elicits multiple biochemical and biophysical responses and therefore requires an appropriate control network. Mitogen-activated protein kinase (MAPK) signalling pathways constitute a network of phosphorylation cascades that link cellular stress to changes in transcriptional activity. MAPK cascades are divided into four major subfamilies, including extracellular signal-regulated kinases 1 and 2, p38 MAPK, c-Jun $\mathrm{NH}_{2}$-terminal kinase and extracellular signal-regulated kinase 5. The present review will present the current understanding of parallel MAPK signalling in human skeletal muscle in response to exercise and muscle contraction, with an emphasis on identifying potential signalling mechanisms responsible for changes in gene expression.
\end{abstract}

Mitogen-activated protein kinase: Skeletal muscle: Exercise

Exercise training has numerous beneficial effects, including glucose homeostasis and enhanced insulin sensitivity (Zierath \& Wallberg-Henriksson, 1992). Examples of exercise-induced changes in skeletal muscle include an isoform switch of myofibrillar components, increased capillary density, increased protein expression of key genes important for metabolic control, increased membrane transport processes and improved sensitivity to hormones such as insulin (Widegren et al. 2001). Thus, physical exercise is a very complex physiological stimulus that challenges multiple biochemical and biophysical mechanisms governing cellular function, and therefore requires an appropriate control network.

Growth factors, cytokines, cellular stress and muscle contraction or exercise lead to changes in the activity of various members of the mitogen-activated protein kinase (MAPK) family. MAPK signalling cascades can be divided into diverse pathways mediated through extracellular signal-regulated kinases (ERK) 1 and 2 (ERK1/2), p38 MAPK and c-Jun $\mathrm{NH}_{2}$-terminal kinases (JNK; Widegren et al. 2001). Since MAPK signalling pathways constitute a well-established network that couples cellular stress to changes in transcriptional activity, exercise traininginduced changes in gene expression may occur in response to increased signal transduction through this cascade. The present review will present the current understanding of MAPK signalling in human skeletal muscle in response to exercise, with an emphasis placed on identifying potential signalling mechanisms responsible for changes in gene expression.

\section{Mitogen-activated protein kinase signalling}

MAPK can be divided into four major subfamilies (Johnson \& Lapadat, 2002; Fig. 1): ERK1/2; p38 MAPK; JNK; ERK5. ERK5 is activated in response to oxidative stress, hyperosmosis and growth factor stimulation (Abe et al. 1996; Pearson et al. 2001). However, since little is known about the regulation of ERK5 in skeletal muscle, this MAPK will not be further discussed. While MAPK signalling can be separated into distinct parallel pathways, these cascades are somewhat conserved and have similar kinase modules (Fig. 2). MAPK are activated through phosphorylation by specific upstream MAPK kinases, which are activated by upstream MAPK kinase kinases. Activation of MAPK kinase kinase in response to external signals, such as growth hormones and cellular stresses, is dependent on specific interaction with receptors,

\footnotetext{
Abbreviations: CREB, cAMP-response element-binding protein; ERK, extracellular signal-related kinase; ERK1/2, ERK 1 and 2; JNK, c-Jun NH 2 terminal kinase; MAPK, mitogen-activated protein kinase; MAPKAP-K, MAPK-activated protein kinase; MEF, myocyte-enhancing factor; MSK1 and 2, mitogen- and stress-activated protein kinase; p90rsk, p90 ribosomal S6 kinase.

*Corresponding author: Dr Juleen R. Zierath, fax +46 83354 36, email Juleen.Zierath@fyfa.ki.se
} 


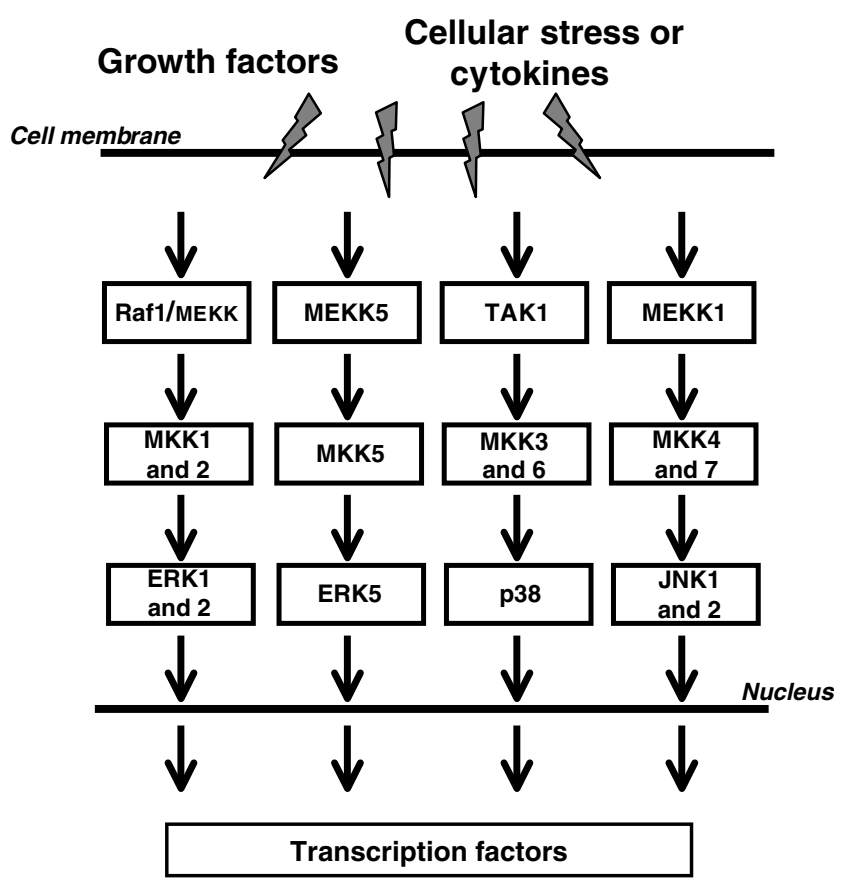

Fig. 1. Schematic diagram illustrating mitogen-activated protein kinase (MAPK) modules. Extracellular signal-related kinase (ERK), c-Jun $\mathrm{NH}_{2}$-terminal kinase (JNK) and p38 MAPK (p38) signalling pathways described in cultured cell systems. MEKK, MAPK/ERK kinase; MKK, MAPK kinase; TAK, transforming growth factor $\beta$-activated kinase; Raf1, serine/threonine kinase.

GTP-binding proteins or other kinases. MAPK phosphorylate diverse substrates, including transcription factors and co-activators localized in the cytoplasm or nucleus, thereby forming a basis for the regulation of transcriptional events, mitosis, cell growth and apoptosis. MAPK have also been implicated in a wide array of cellular events such as inflammation and carbohydrate metabolism, highlighting their profound influence on cellular physiology. Relevant to the present review is the observation that exercise leads to the activation of at least three MAPK signalling pathways, i.e. ERK1/2, p38 MAPK and JNK, in skeletal muscle (Zierath, 2002). Thus, MAPK signalling cascades form a candidate network of signal transducers that may regulate changes in gene expression in response to exercise.

\section{Extracellular signal-related kinases 1 and 2 mitogen-activated protein kinase pathway}

ERK1/2, the first of the identified MAPK isoforms, are activated in response to mitogenic stimuli, including growth factors, via receptor tyrosine kinases, G-proteincoupled receptors, protein kinase $\mathrm{C}$ and cellular stress (van Biesen et al. 1996; Schonwasser et al. 1998). Using cell-based systems and in vitro kinase assays, several downstream substrates of ERK1/2 kinase have been identified (Brunet \& Pouyssegur, 1997), including p90 ribosomal S6 kinase (p90rsk, also known as MAPKactivated protein kinase (MAPKAP-K) 1; Zhao et al. 1996) and mitogen- and stress-activated kinases (MSK) 1 and 2

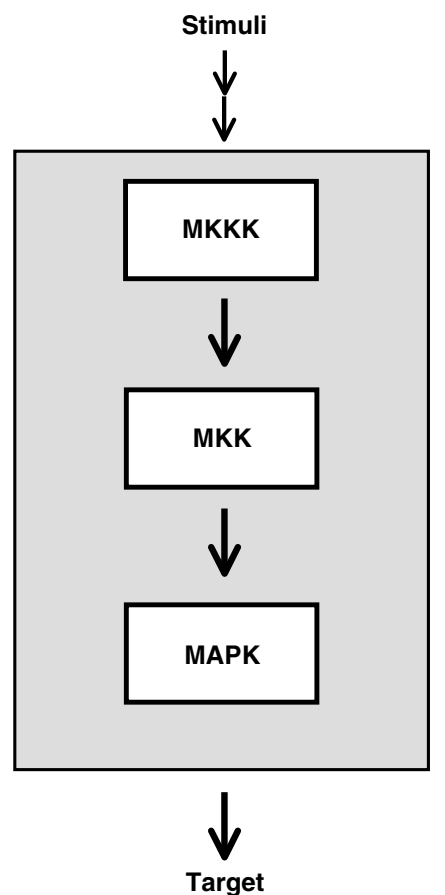

Fig. 2. Mitogen-activated protein kinase (MAPK) module. A core module of the MAPK pathway consists of three kinases: MAPK kinase kinase (MKKK); MAPK kinase (MKK); MAPK; these kinases undergo sequential phosphorylation.

(Deak et al. 1998). Activated p90rsk phosphorylates several transcription factors including Elk and the cAMPresponse element-binding protein (CREB) and the activating protein 1 family of c-Jun, c-fos and activating transcription factor (Pearson et al. 2001). Since members of the ERK pathway are widely expressed in skeletal muscle, one obvious question to address is whether physiological stress, such as muscle contraction through exercise training, elicits ERK signalling.

Activation of the ERK1/2 pathway has been reported in human skeletal muscle after acute cycling exercise (Widegren et al. 1998, 2000; Yu et al. 2003) and marathon running ( $\mathrm{Yu}$ et al. 2001). Using a one-leg cycle exercise protocol $\left(70 \% \mathrm{~V}_{\mathrm{O}_{2} \max }\right)$, ERK1/2 phosphorylation has been shown to occur exclusively in the exercised leg (Widegren et al. 1998). Thus, local rather than systemic effects of muscle contraction appear to induce ERK1/2 activation. This observation has been confirmed in vitro, since contraction of isolated rat skeletal muscle is sufficient to elicit ERK1/2 phosphorylation (Ryder et al. 2000; Wretman et al. 2000, 2001). These results suggest that contraction per se is sufficient to activate ERK1/2.

In moderately-trained subjects ERK1/2 phosphorylation occurs early in the exercise bout (after $10 \mathrm{~min}$ of submaximal cycling), with the greatest effect observed after approximately $30 \mathrm{~min}$ (Widegren et al. 1998). ERK1/2 phosphorylation is maintained throughout the exercise bout, with phosphorylation rapidly diminishing on cessation of exercise. Exercise and contraction have been reported to activate MAPK/ERK1/2 (Aronson et al. 1997b; Widegren et al. 2000) and serine/threonine kinase (Aronson et al. 1997b) in human skeletal muscle. Interestingly, 
exercise-induced phosphorylation of MAPK/ERK1/2 and ERK1/2 occurs in an intensity-dependent manner, as ERK1/2 phosphorylation is greater in high $(75 \%$ of one-leg $\mathrm{V}_{\mathrm{O}_{2} \max }$ )-intensity $v$. low (40\% of one-leg $\mathrm{V}_{\mathrm{O}_{2} \max }$ )intensity cycling exercise (Widegren et al. 2000). However, the effects of exercise on ERK1/2 phosphorylation are not limited to untrained subjects. In highly-trained athletes acute exercise also leads to ERK1/2 phosphorylation (Yu et al. 2003).

Exercise has also been shown to activate downstream targets of ERK1/2, including p90rsk and MSK1 and 2 (Krook et al. 2000; Yu et al. 2001). Activation of MSK1 and 2 is rapid and limited to the exercising muscle. Thus, activation of MSK1 and 2 in response to exercise appears to occur primarily via the ERK1/2-dependent pathway (Krook et al. 2000). While the physiological importance of MAPK activation can be revealed in human studies, it is not possible to draw definitive conclusions about the precise interaction between identified MAPK substrates and the upstream regulators. In order to determine substrate specificity in MAPK signalling cascades an ex vitro system has been employed to achieve contraction (electrical stimulation) of isolated rodent skeletal muscle, combined with the application of commonly-used chemical inhibitors of ERK (Cuenda et al. 1995) and p38 MAPK (Alessi et al. 1995); PD98059 (MAPK/ERK1 inhibitor) and SB203580 (p38 MAPK inhibitor) respectively. In isolated rat skeletal muscle specific inhibition of ERK1/2 by PD98059 completely abolishes contraction-mediated p90rsk activity (Ryder et al. 2000). In contrast, activation of MSK1 requires simultaneous activation of both ERK1/2 and p38 MAPK (Ryder et al. 2000). While there may be some differences in MSK signalling in response to exercise and electrical stimulation, contraction-induced induction of p90rsk and MAPKAP-K2 is likely to occur largely via separate pathways, reflecting ERK and p38 MAPK stimulation respectively, whereas MSK1 induction requires simultaneous activation of both ERK and p38 MAPK.

\section{p38 mitogen-activated protein kinase pathway}

Studies of MAPK are complicated by the presence of parallel signalling pathways that can undergo simultaneous activation. p38 MAPK shares common kinase substrates with ERK1/2, including MAPKAP K-2 and -3, MSK1 and 2 and CREB (Deak et al. 1998; Pearson et al. 2001). Thus, there is a high extent of cross-talk between the ERK and p38 MAPK kinase pathways and specificity of signalling between these two pathways has not been fully resolved. p38 MAPK signal transduction can be mediated by four different isoforms, each of which display differential sensitivity to chemical inhibitors, such that p38 $\alpha$ and $\mathrm{p} 38 \beta$ are inhibited by SB203580, whereas p38 $\delta$ and $\mathrm{p} 38 \gamma$ are not (Goedert et al. 1997). The existence of different p38 isoforms is likely to give specificity in mediating downstream biological responses. Few studies have elucidated isoformspecific roles of $\mathrm{p} 38$ MAPK in skeletal muscle, thus the following discussion will focus on the general role of $\mathrm{p} 38$ MAPK in response to contraction.

In human subjects p38 MAPK is phosphorylated in skeletal muscle in response to cycle exercise (Widegren et al. 1998) and marathon running (Boppart et al. 2000; Yu et al. 2001). In contrast to marked but transient ERK1/2 activation, one-leg cycling exercise leads to a smaller increase but more persistent p38 MAPK activation. p38 MAPK phosphorylation is also increased in the resting leg, indicating the potential influence of a systemic factor (Widegren et al. 1998). Differences in the concentric $v$. eccentric mode of contraction or training status of the subject may influence MAPK signalling. In isolated rat skeletal muscle concentric contractions increase ERK1/2 phosphorylation with no effect on p38 MAPK, whereas eccentric contractions increase phosphorylation along both kinase cascades (Wretman et al. 2001). In human subjects one session of eccentric contractions during leg press resistance exercise is associated with an increase in ERK1/2 phosphorylation, with no effect on p38 MAPK phosphorylation (Williamson et al. 2003). With exhaustive exercise, both ERK and p38 MAPK pathways are likely to be invoked. Alternatively, the divergent effects on MAPK signalling may be related to the training status of the subjects. Interestingly, exercise-induced signalling responses for p38 MAPK have been shown to be greater in untrained men, even at the same relative cycling exercise intensity, even though the trained and untrained subjects exercised at approximately the same relative intensity (approximately $85-90 \%$ of $\mathrm{V}_{\mathrm{O}_{2} \max }$; $\mathrm{Yu}$ et al. 2003). Thus, skeletal muscle from previously-highlytrained individuals appears to require a greater stimulus to activate signal transduction via p38 MAPK, indicating an extent of training-induced adaptation along MAPK pathways. The full extent of adaptation along these pathways may be revealed through future studies of MAPK signalling in untrained subjects before and after intensive training periods.

Cycling exercise and marathon running also activate MAPKAP-K2, a known downstream target of p38 MAPK (Krook et al. 2000; Yu et al. 2001, 2003). Consistent with this observation, in vitro inhibition of p38 MAPK by SB203580 in rat skeletal muscle abolishes activation of MAPKAP-K2 by in vitro electrically-stimulated muscle contraction (Ryder et al. 2000). Other downstream targets of p38 MAPK include transcription factors such as Elk1 and myocyte-enhancing factor (MEF) 2, whereby p38 MAPK directly phosphorylates nuclear substrates and augments their activity (Chen et al. 2001). The direct targets of p38 MAPK in human skeletal muscle in response to exercise are currently unknown. In cultured myoblasts evidence has been presented that MEF2 binding is necessary for p38 MAPK regulation of muscle creatine kinase promoter-driven reporter gene expression (Zetser et al. 1999). Furthermore, Yu et al. (2001) have recently provided evidence that MEF2 sequence-specific DNA binding is increased in human skeletal muscle after completion of marathon running. While the direct link between p38 MAPK and exercise-mediated MEF2 binding remains to be demonstrated, these findings have important implications, as MEF2-binding sequences are present in the promoter region of a vast array of muscle specific genes that are critical for metabolic and physiological processes. Examples of genes having MEF2-binding sites include GLUT4, creatine kinase, AMP deaminase, myoglobin, 
myosin (heavy-light chains), dystrophin, $M y o D$ and $c-J u n$ (Black \& Olson, 1998).

\section{c-Jun $\mathrm{NH}_{2}$-terminal kinase pathway}

The differential response of the MAPK signalling pathways to physical exercise may be related to the type of cellular stress induced during the exercise bout or the differences in the concentric $v$. eccentric mode of contraction. For example, signal transduction through the JNK pathway in human skeletal muscle is highly influenced by injurypromoting exercise, with greater effects noted after eccentric $v$. concentric exercise (Boppart et al. 1999), suggesting that the level of activation is somewhat dependent on the nature of exercise. Stress-activated protein kinase/ERK kinase 1, a potent activator of JNK, is also activated during one-leg cycle ergometry (Widegren et al. 1998). Activation of JNK in response to skeletal muscle contraction is associated with a rapid induction of the early response genes $c$-Jun and $c$-fos, as evidenced by increased mRNA levels (Aronson et al. 1997a). As transcription factor c-Jun is a downstream target of JNK, the JNK pathway is implicated in mediating the mitogenic response of skeletal muscle to exercise.

\section{Mitogen-activated protein kinase cascades and transcriptional regulation}

The downstream targets along the MAPK pathways that mediate transcriptional events have been delineated from studies in cultured cells and, thus, require validation in human skeletal muscle. Activated p90rsk has been proposed to phosphorylate several transcription factors, including Elk1 and CREB (Widegren et al. 2001). In 293 cells activated MSK1 is localized in the nucleus and phosphorylates the transcription factor CREB at Ser ${ }^{133}$ (Deak et al. 1998). Yet, exercise is not associated with increased CREB phosphorylation in the working skeletal muscle; CREB phosphorylation in skeletal muscle after exercise is either unchanged (Widegren et al. 2000) or repressed (Widegren et al. 1998). Thus, while MSK1 is a highly efficient CREB kinase in vitro, it does not appear to be linked with CREB phosphorylation in skeletal muscle after exercise.

Another plausible link between MAPK substrates and transcriptional events is through the regulation of nucleosomal responses through the rapid serine phosphorylation of histone $\mathrm{H} 3$ that occurs concomitantly with immediateearly gene induction in response to a wide variety of stimuli. MAPK substrates mediate alterations in the chromatin environment of specific genes by direct phosphorylation and/or acetylation of nucleosomal and chromatin proteins, thereby directly linking the MAPK pathway to induction of immediate-early response gene expression (Thomson et al. 1999b). Alterations in the nucleosome and chromatin structure can occur through phosphorylation of histone $\mathrm{H} 3$, which is associated with MAPK signalling to p90rsk (Nebreda \& Gavin, 1999; Sassone-Corsi et al. 1999) and MSK1 (Thomson et al. 1999a). Previous studies in 293 cells provide evidence that the nuclear protein histone H3/HMG14 is a physiological substrate of MSK1
(Thomson et al. 1999a). Intense cycling exercise is associated with an increase in histone $\mathrm{H} 3$ phosphorylation in skeletal muscle ( $\mathrm{Yu}$ et al. 2003). While the extent of the increase in histone $\mathrm{H} 3$ phosphorylation is similar for trained and control subjects, the resting histone $\mathrm{H} 3$ phosphorylation is slightly greater in trained subjects. The physiological significance of the increased resting histone $\mathrm{H} 3$ phosphorylation in trained subjects is unknown, but may be a response related to chronic exercise training. Although studies in human skeletal muscle cannot directly link exercise-induced responses of MAPK signalling to changes in immediate-early genes, these correlative studies provide evidence to suggest MSK1 and histone H3 mediate changes in immediate-early genes. Other links between the MAPK signalling pathway and translation initiation of protein synthesis have been suggested via MAPK integrating kinase-1, a substrate of both ERK and p38 MAPK (Raught \& Gingras, 1999); however, this link has yet to be confirmed in skeletal muscle in response to exercise.

Exercise-induced MAPK signalling pathways are now beginning to be identified, with defined links to specific targets that are involved in regulation of transcription machinery within skeletal muscle (Fig. 3). Clearly, one of the greatest challenges of the exercise physiologist will be to further define the specific transcriptional machinery mediating changes in gene expression in skeletal muscle in response to exercise training. The interaction between MAPK pathways and downstream targets is likely to differ from that identified in cultured cells. Thus, pathway validation in skeletal muscle in response to exercise is required.

\section{Summary and future directions}

Muscle contraction through physical exercise is a potent activator of the MAPK signalling cascade. The activation profile of ERK1/2, p38 MAPK and JNK in human skeletal muscle during exercise is distinctively divergent and depends on the nature of the exercise. Furthermore, training status also influences the extent of MAPK activation. Thus, future studies designed to determine the effects of different modes of exercise in subjects with varied levels of fitness on MAPK signalling are warranted. More importantly, the identification of specific roles of each MAPK pathway in mediating exercise-induced mitogenic adaptations remains to be elucidated. As downstream targets of MAPK include numerous transcription factors, activation of the MAPK signalling pathway is likely to mediate exercise-induced changes in gene expression in skeletal muscle. However, the direct link between exercise-mediated MAPK signalling and changes in the expression of specific genes remains to be delineated. Thus, the link between exercise-induced MAPK signalling and changes in specific genes or gene patterns will be important to resolve. Through in vitro studies, using electrically-stimulated contraction protocols to mimic exercise in isolated rodent skeletal muscle, coupled with the use of specific MAPK inhibitors, the specific roles of the parallel MAPK pathways and the specific downstream substrates in mediating exerciseinduced changes in gene expression can be determined. Functional genomics also offers a means for determining 


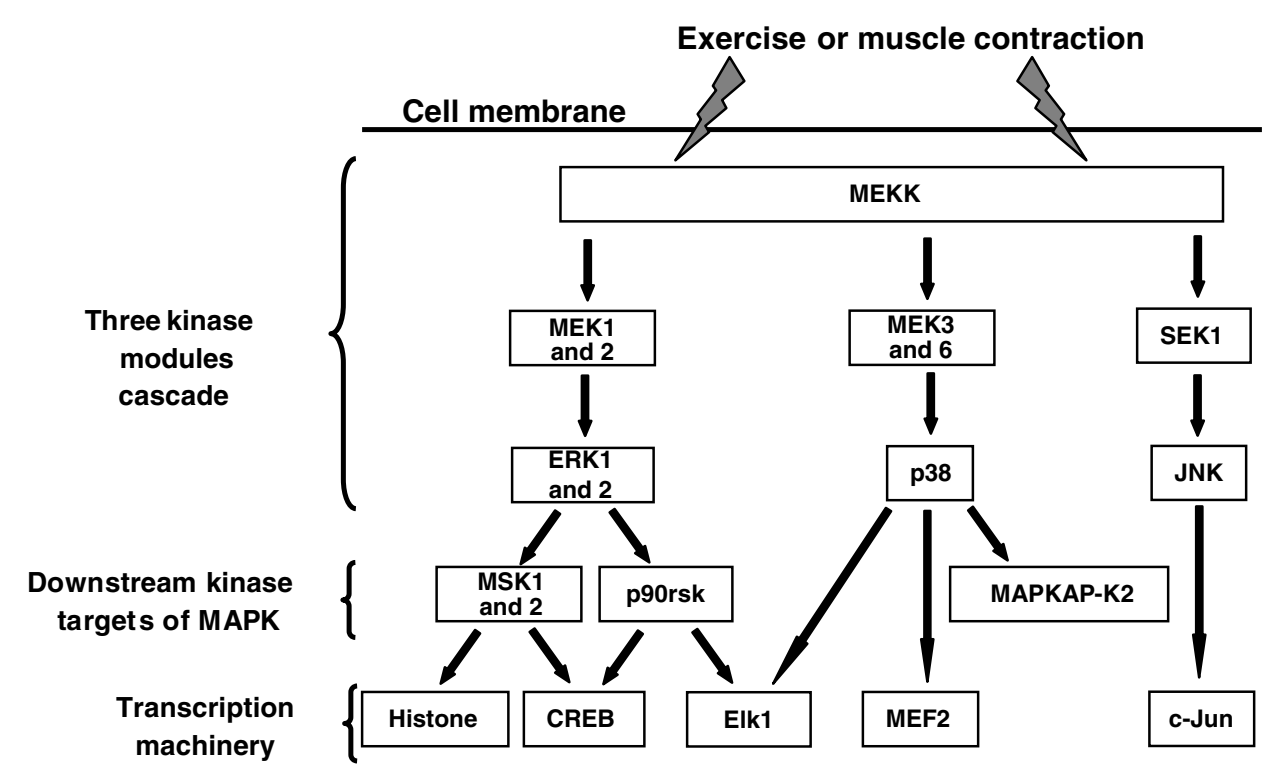

Fig. 3. Putative exercise-mediated mitogen-activated protein kinase (MAPK) signalling cascades in skeletal muscle. MEK, MAPK/ERK; MEKK, MEK kinase; SEK, stress-activated protein kinase/ERK kinase; ERK, extracellular signal-related kinase; p38, p38 MAPK; MAPKAP-K2, MAPK-activated protein kinase 2; JNK, c-Jun $\mathrm{NH}_{2}$-terminal kinase; $\mathrm{MSK}$, mitogen- and stress-activated kinase; p90rsk, p90 ribosomal S6 kinase; CREB, cAMP response element-binding protein; MEF, myocyteenhancing factor.

the physiological role of MAPK in mediating exerciseinduced changes in gene expression. Mouse gene knockout models of MAPK kinase and MAPK are available and can be used for this purpose. The use of knock-out models circumvents the lack of isoform-specific inhibition of the MAPK by pharmacological inhibitors. However, in some cases, such as the gene knock-out of the p38 $\alpha$ isoform, embryonic lethality will hamper further investigation of the functional role of a particular kinase isoform. The temporal and tissue-specific knock-out of specific target gene in mouse models can be achieved by the application of the Cre/loxP recombination system (a commonly used tool to alter the mouse genome in a conditional manner by deletion or inversion of loxP-flanked DNA segments). Finally, results from studies performed using animal models should be confirmed in human subjects. Expression profiling, performed on skeletal muscle biopsy obtained after exercise, offers a powerful strategy for revealing the genes that are regulated by exercise. Gene and/or protein array technology will offer a high-throughput means of identifying targets that are differentially controlled by exercise on a transcriptional or translational level. The goal should be to develop a defined network of cellular signalling cascades controlling transcriptional responses in skeletal muscle in response to exercise.

\section{Acknowledgements}

The authors' laboratory is supported by grants from the Swedish Medical Research Council, the Swedish Diabetes Association, Novo-Nordisk Foundation, Marcus and Amalia Wallenberg's Foundation, Torsten and Ragnar
Söderberg's Foundation and the Foundation for Scientific Studies of Diabetology.

\section{References}

Abe JI, Kusuhara M, Ulevitch RJ, Berk BC \& Lee JD (1996) Big mitogen-activated protein kinase 1(BMK1) is a redox-sensitive kinase. Journal of Biological Chemistry 271, 16586-16590.

Alessi DR, Cuenda A, Cohen P, Dudley DT \& Saltiel AR (1995) PD 098059 is a specific inhibitor of the activation of mitogenactivated protein kinase in vitro and in vivo. Journal of Biological Chemistry 270, 27489-27494.

Aronson D, Dufresne SD \& Goodyear LJ (1997a) Contractile activity stimulates the c-Jun NH2-terminal kinase pathway in rat skeletal muscle. Journal of Biological Chemistry 272, 25636-25640.

Aronson D, Violan MA, Dufresne SD, Zangen D, Fielding RA \& Goodyear LJ (1997b) Exercise stimulates the mitogenactivated protein kinase pathway in human skeletal muscle. Journal of Clinical Investigation 99, 1251-1257.

Black BL \& Olson EN (1998) Transcriptional control of muscle development by myocyte enhancer factor-2 (MEF2) proteins. Annual Review of Cell Developmental Biology 14, 167-196.

Boppart MD, Aronson D, Gibson L, Roubenoff R, Abad LW, Bean J, Goodyear LJ \& Fielding RA (1999) Eccentric exercise markedly increases c-Jun $\mathrm{NH}_{2}$-terminal kinase activity in human skeletal muscle. Journal of Applied Physiology 87, $1668-1673$

Boppart MD, Asp S, Wojtaszewski JFP, Fielding RA, Mohr T \& Goodyear LJ (2000) Marathon running transiently increases c-Jun $\mathrm{NH}_{2}$-terminal kinase and $\mathrm{p} 38 \gamma$ activities in human skeletal muscle. Journal of Physiology (London) 526, 663-669.

Brunet A \& Pouyssegur J (1997) Mammalian MAP kinase modules: How to transduce specific signals. Essays in Biochemistry 32, 1-16. 
Chen Z, Gibson TB, Robinson F, Silvestro L, Pearson G, Xu B, Wright A, Vanderbilt C \& Cobb MH (2001) MAP kinases. Chemical Reviews 101, 2449-2476.

Cuenda A, Rouse J, Doza YN, Meier R, Cohen P, Gallagher T-F, Young PR \& Lee JC (1995) SB 203580 is a specific inhibitor of a MAP kinase homologue which is stimulated by cellular stresses and interleukin-1. FEBS Letters 364, 229-233.

Deak M, Clifton A, Lucocq L \& Alessi D (1998) Mitogen- and stress-activated protein kinase-1 (MSK1) is directly activated by MAPK and SAPK2/p38, and may mediate activation of CREB. EMBO Journal 15, 6552-6563.

Goedert M, Cuenda A, Craxton M, Jakes R \& Cohen P (1997) Activation of the novel stress-activated protein kinase SAPK4 by cytokines and cellular stresses is mediated by SKK3 (MKK6); comparison of its substrate specificity with that of other SAP kinases. EMBO Journal 16, 3563-3571.

Johnson GL \& Lapadat R (2002) Mitogen-activated protein kinase pathways mediated by ERK, JNK, and p38 protein kinases. Science 298, 1911-1912.

Krook A, Widegren U, Jiang XJ, Henriksson J, WallbergHenriksson H, Alessi D \& Zierath JR (2000) Effects of exercise on mitogen- and stress-activated kinase signal transduction in human skeletal muscle. American Journal of Physiology 279, R1716-R1721.

Nebreda AR \& Gavin AC (1999) Cell survival demands some Rsk. Science 286, 1309-1310.

Pearson G, Robinson F, Beers Gibson T, Xu BE, Karandikar M, Berman K \& Cobb MH (2001) Mitogen-activated protein (MAP) kinase pathways: Regulation and physiological functions. Endocrine Reviews 22, 153-183.

Raught B \& Gingras AC (1999) eIF4E activity is regulated at multiple levels. International Journal of Biochemistry and Cell Biology 31, 43-57.

Ryder JW, Fahlman R, Wallberg-Henriksson H, Alessi DR, Krook A \& Zierath JR (2000) Effect of contraction on mitogen-activated protein kinase signal transduction in skeletal muscle: Involvement of the mitogen- and stress-activated protein kinase 1. Journal of Biological Chemistry 275, 1457-1462.

Sassone-Corsi P, Mizzen CA, Cheung P, Crosio C, Monaco L, Jacquot S, Hanauer A \& Allis CD (1999) Requirement of Rsk-2 for epidermal growth factor-activated phosphorylation of histone H3. Science 285, 886-891.

Schonwasser DC, Marais RM, Marshall CJ \& Parker PJ (1998) Activation of the mitogen-activated protein kinase/extracellular signal-regulated kinase pathway by conventional, novel, and atypical protein kinase $\mathrm{C}$ isotypes. Molecular and Cellular Biology 18, 790-798.

Thomson S, Clayton AL, Hazzalin CA, Rose S, Barratt MJ \& Mahadevan LC (1999a) The nucleosomal response associated with immediate-early gene induction is mediated via alternative MAP kinase cascades: MSK1 as a potential histone H3/HMG-14 kinase. EMBO Journal 18, 4779-4793.

Thomson S, Mahadevan LC \& Clayton AL (1999b) MAP kinasemediated signalling to nucleosomes and immediate-early gene induction. Seminars in Cell and Developmental Biology 10 205-214.

van Biesen T, Hawes BE, Raymond JR, Luttrell LM, Koch WJ \& Lefkowitz RJ (1996) G(o)-protein $\alpha$-subunits activate mitogenactivated protein kinase via a novel protein kinase C-dependent mechanism. Journal of Biological Chemistry 271, 1266-1269.

Widegren U, Jiang XJ, Krook A, Chibalin AV, Björnholm M, Tally M, Roth RA, Henriksson J, Wallberg-Henriksson H \& Zierath JR (1998) Divergent effects of exercise on metabolic and mitogenic signaling pathways in human skeletal muscle. FASEB Journal 12, 1379-1389.

Widegren U, Ryder JW \& Zierath JR (2001) Mitogen-activated protein kinase (MAPK) signal transduction in skeletal muscle: Effects of exercise and muscle contraction. Acta Physiologica Scandinavica 172, 227-238.

Widegren U, Wretman C, Lionikas A, Westerblad H \& Henriksson J (2000) Influence of exercise intensity on ERK/ MAP kinase signalling in human skeletal muscle. Pflugers Archives European Journal of Physiology 441, 317-322.

Williamson D, Gallagher P, Harber M, Hollon C \& Trappe S (2003) Mitogen-activated protein kinase (MAPK) pathway activation: effects of age and acute exercise on human skeletal muscle. Journal of Physiology (London) 547, 977-987.

Wretman C, Lionikas A, Widegren U, Lannergren J, Westerblad H \& Henriksson J (2001) Effects of concentric and eccentric contractions on phosphorylation of MAPKerk1/2 and MAPKp38 in isolated rat skeletal muscle. Journal of Physiology (London) 535, 155-164.

Wretman C, Widegren U, Lionikas A, Westerblad H \& Henriksson J (2000) Differential activation of mitogen-activated protein kinase signalling pathways by isometric contractions in isolated slow- and fast-twitch rat skeletal muscle. Acta Physiologica Scandinavica 170, 45-49.

Yu M, Blomstrand E, Chibalin AV, Krook A \& Zierath JR (2001) Marathon running increases ERK1/2 and p38 MAP kinase signalling to downstream targets in human skeletal muscle. Journal of Physiology (London) 536, 273-282.

Yu M, Stepto NK, Chibalin AV, Fryer LGD, Carling D, Krook A Hawley JA \& Zierath JR (2003) Metabolic and mitogenic signal transduction in human skeletal muscle after intense cycling exercise. Journal of Physiology (London) 546, 327-335.

Zetser A, Gredinger E \& Bengal E (1999) p38 mitogen-activated protein kinase pathway promotes skeletal muscle differentiation. Participation of the MEF2c transcription factor. Journal of Biological Chemistry 274, 5193-5200.

Zhao Y, Bjorbaek C \& Moller DE (1996) Regulation and interaction of pp90(RSK) isoforms with mitogen-activated protein kinase. Journal of Biological Chemistry 271, 29773-29779.

Zierath JR (2002) Exercise effects of muscle insulin signaling and action. Exercise training-induced changes in insulin signaling in skeletal muscle. Journal of Applied Physiology 93, 773-781.

Zierath JR \& Wallberg-Henriksson H (1992) Exercise training in obese diabetic patients. Sports Medicine 14, 171-189. 